Revista internacional de fronteras, territorios y regiones / International Journal of Borders, Territories and Regions

FRONTERA NORTE VOL. 32, ART. 10, 2020

https://doi.org/10.33679/rfn.v1i1.1973

\title{
Acuerdos y conflictos en dos localidades fronterizas chuj entre México y Guatemala
}

Agreements and Conflicts in Two Chuj Border Towns Between Mexico and Guatemala

Ludivina Mejía González ${ }^{1}$

\section{RESUMEN}

Este artículo describe etnográficamente algunos acuerdos y conflictos que se presentan en un fragmento fronterizo compartido por dos comunidades chuj que viven en El Quetzal, ubicado en territorio guatemalteco, y en Tziscao, localizado en México. Este trabajo se propone reflexionar y analizar las formas en que las comunidades fronterizas sostienen acuerdos y conflictos como fenómenos inherentes de las relaciones sociales y las dinámicas cotidianas. Se contextualiza la conformación del territorio fronterizo a partir de la delimitación de la frontera geopolítica delimitada en 1882, y se muestran los accesos diferenciados a bienes y servicios que son posibles a partir de la condición nacional de sus habitantes. En este proceso surge una serie de acuerdos y conflictos vinculados con el agua doméstica, el comercio, las actividades turísticas y el cruce por la frontera de poblaciones guatemaltecas hacia México. Finalmente, se observa que en medio de los conflictos existen relaciones socioculturales de continuidad e historias de reciprocidad que sostienen las relaciones sociales del grupo cultural chuj.

Palabras clave: 1. acuerdos, 2. conflictos, 3. frontera, 4. México, 5. Guatemala.

\section{ABSTRACT}

This article ethnographically describes agreements and conflicts present in a border fragment shared by two Chuj communities: El Quetzal, located in Guatemalan territory, and Tziscao in Mexico. This paper intends to reflect and analyze how border communities maintain agreements and conflicts as inherent phenomena of daily social and dynamic relationships. The conformation of the border territory is contextualized from the delimitation of the geopolitical border in 1882, also differentiated accesses to material goods and services, which are possible from the national condition of its inhabitants, are presented. In this process, a series of agreements and conflicts related to domestic water, commerce, tourism activities, and the border crossing of the Guatemalan population to Mexico arise. Finally, it is observed that amid conflicts existing socio-cultural relations of continuity and stories of reciprocity sustain the social relations of the Chuj cultural group.

Keywords: 1. agreements, 2. conflicts, 3. border, 4. Mexico 5. Guatemala.

Fecha de recepción: 3 de marzo de 2019

Fecha de aceptación: 23 de septiembre de 2019

Publicación en web: 1 de julio de 2020

\footnotetext{
${ }^{1}$ Centro de Investigaciones y Estudios Superiores en Antropología Social-Sureste, México, ludivina_m2@hotmail.com, https://orcid.org/0000-0001-6767-3734
} 
2 Acuerdos y conflictos en dos localidades fronterizas Chuj entre México y Guatemala Mejía González, L.

\section{INTRODUCCIÓN}

Los acuerdos y conflictos que viven las comunidades fronterizas del sur de México se explican, de alguna manera, como dos fenómenos inherentes de las relaciones sociales y las dinámicas cotidianas. Desde luego, las relaciones sociales tienen una multitud de formas, no solo con la disputa y el conflicto como reveladores de la vida social, sino también con el ocultamiento, la aceptación de las contradicciones y la amnesia, como formas muy extendidas de la relación social que viven y enfrentan las comunidades que comparten un territorio (Marié, 2015).

En la frontera México-Guatemala se pueden observar ambos fenómenos a la vez -acuerdos y conflictos-, y por lo tanto, es necesario abordarlos de manera conjunta. Estas relaciones sociales históricas existentes se sostienen en las tensiones que Castillo (1994, 1999) denomina proximidad y distancia. El autor afirma que en la frontera entre México y Guatemala existe proximidad no solo limítrofe y geográfica, sino también en las condiciones de vida, la comunidad lingüística y las pautas socioculturales. El límite geopolítico es el que "separa" y crea la distancia; a la vez, este es rebasado por las dinámicas sociales que se gestan en la vecindad de las comunidades.

Es importante decir que no todos los conflictos se presentan de la misma manera en toda la frontera sur, pues cada fragmento fronterizo entre México y Guatemala tiene sus propias particularidades. Sin embargo, en esta frontera de México podemos encontrar muchas comunidades vecinas que viven en escenarios similares como el que acontece entre El Quetzal, ubicado en territorio guatemalteco, y Tziscao, localizado en México. Aunque históricamente, ambas comunidades fronterizas han establecido una serie de convenios, acuerdos y arreglos, en las dinámicas cotidianas surgen diferenciaciones, contradicciones e intereses que llevan a una serie de tensiones y conflictos.

Por supuesto que estas relaciones complejas se entienden y se explican a partir de los procesos históricos que han conformado el territorio fronterizo. La delimitación de la frontera nacional de 1882 "separó" comunidades, y a la vez marcó una diferenciación entre los pobladores por el hecho de formar parte de una nacionalidad diferente.

Kauffer Michel (2011) afirma que a partir de la delimitación de la frontera geopolítica entre México y Guatemala que se dio a finales del siglo XIX, quedaron una serie de conflictos y asimetrías. De la misma manera, Castillo (2002) plantea que estos fragmentos fronterizos son espacios de continuidad, de afinidades, semejanzas que adquieren dimensiones transfronterizas.

Las localidades de Tziscao, ubicada en el sur sureste del estado de Chiapas, y de El Quetzal, en la zona noroccidental del departamento de Huehuetenango, ocupan un fragmento fronterizo entre México y Guatemala, a la altura del denominado Vértice de Santiago (Ver mapa 1). Esta área geográfica está caracterizada por su riqueza hídrica; en ella se encuentra el Sistema Lagunar de Montebello, conformado por 59 cuerpos de agua, la mayor parte de 
ellos están del lado mexicano y la sección de una laguna se encuentra del lado guatemalteco, llamada Laguna Binacional, que está dividida por la frontera geopolítica.

Mapa 1. Unidad de análisis territorial en la frontera México-Guatemala

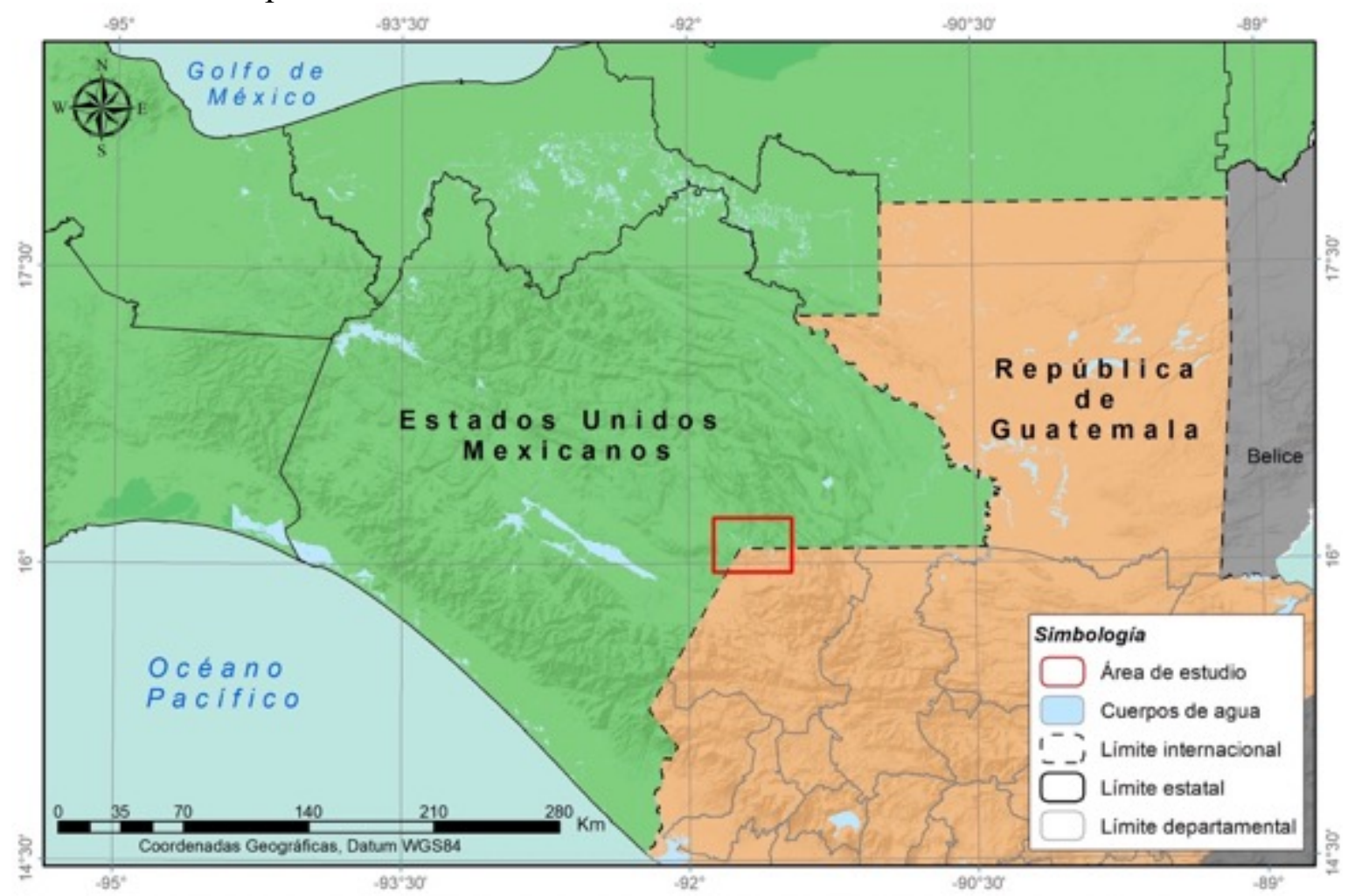

Fuente: Capas temáticas de Instituto Nacional de Estadística y Geografía (Inegi, 2010), Ministerio de Agricultura, Ganadería y Alimentación (MAGA, 2005). Elaboró César Octavio Sánchez Garay.

El Quetzal y Tziscao son comunidades vecinas, separadas por la línea fronteriza. Para cruzar de El Quetzal hacia la carretera federal del lado mexicano, sus pobladores tienen que recorrer un tramo de aproximadamente dos kilómetros, pasando por tierras que corresponden a Tziscao. La lejanía del El Quetzal de centros comerciales y económicos del lado guatemalteco, y la falta de servicios públicos de salud, energía eléctrica, educación y medios de comunicación y transporte, ha llevado a su población a negociar, acordar y convenir con la comunidad de Tziscao.

Durante el 2008 se llevó a cabo una serie de acuerdos y arreglos en torno al agua para uso doméstico de algunas familias de Tziscao; a cambio de esto, las autoridades de Tziscao ofrecieron a los pobladores de El Quetzal servicios de salud y educación, así como permisos para el tránsito de vehículos para cruzar desde la línea divisoria hacia la carretera federal fronteriza mexicana. Sin embargo, por esos mismos años aumentó la presencia del turismo -que llegaba no solo a admirar el sistema lagunar, sino también para cruzar la frontera y visitar Guatemala-, y en ambos lados creció el número de comercios. La competencia y el control del territorio turístico llevaron a la fragmentación de los convenios y, en consecuencia, aparecieron tensiones y conflictos. 
4 Acuerdos y conflictos en dos localidades fronterizas Chuj entre México y Guatemala Mejía González, L.

Por supuesto que el conflicto abierto fue el desenlace de periodos de ocultamiento y simulaciones, porque en el 2012 los pobladores de Tziscao obstaculizaron el paso de vehículos de Guatemala a México. En esa ocasión, los pobladores se aprovecharon de su atributo y condición de ser nacionales mexicanos para imponerse ante sus vecinos. La proximidad se vio trastocada cuando los intereses de ambos grupos se pusieron en juego, y el papel de la frontera límite surgió para marcar la distancia.

Es en ese contexto en el que se intenta reflexionar y analizar de qué manera las comunidades fronterizas sostienen acuerdos y conflictos como fenómenos inherentes de las relaciones sociales y de las dinámicas cotidianas.

El artículo está dividido en tres apartados; en el primero se reflexiona sobre acuerdos y conflictos desde la perspectiva de diversos actores. Posteriormente, se hace referencia al contexto histórico de la conformación de la frontera geopolítica que dividió México y Guatemala, situando a las comunidades El Quetzal y Tziscao. En el tercer apartado se describen los acuerdos, disputas y conflictos que se presentaron a partir de 2008 entre ambas localidades, poniendo atención en cuatro ejes: agua, comercio, turismo y el paso por la frontera; se detalla cómo las relaciones de parentesco disminuyen las tensiones, pues las redes familiares y de trabajo sirven de puentes para la interacción en la cotidianidad y en los procesos de vecindad. Finalmente, daré algunas conclusiones.

\section{¿DE QUÉ MANERA ENTENDER LOS ACUERDOS Y CONFLICTOS COMO PARTE DE LAS RELACIONES SOCIALES?}

Abordar el conflicto como una relación social implica distinguirlo de escenarios de guerra o de ruptura; en ese sentido, el conflicto puede explicarse no solo de manera teórica, sino también empírica e históricamente (Wieviorka, 2010). Marié (2004) propone entender los conflictos como procesos que se dan en las relaciones sociales, que algunas veces se manifiestan o se presentan como de baja intensidad, y pocas veces llegan a mostrarse realmente como conflictos violentos o abiertos, de alta intensidad. El autor refiere que esto se puede explicar por ciertas ambigüedades de la vida social, como cuando los individuos actúan o utilizan la fuerza y la astucia, el paso violento y la negociación. Es decir, los actores sociales recurren a diversas estrategias y utilizan diversos componentes, con manifestaciones que los llevan a actuar de formas paradójicas.

Lederach (1992) también considera importante analizar los conflictos como procesos, porque es ahí en donde se descubre, de alguna manera, la raíz del problema, y no se da por sentado que la causa ya se conoce, e incluso, que puede haber muchos problemas interconectados. El autor menciona que en el epicentro muchas veces subyace la historia del conflicto, que consiste en un proceso histórico-cultural que puede favorecer las relaciones conflictivas; es decir, que es necesario mirar el conflicto de manera integral, enmarcada en una red de relaciones, y no separadamente. 
De acuerdo con Wieviorka (2010), el tema de los conflictos en las relaciones sociales no se reduce al enfrentamiento entre enemigos, sino que también puede ser una relación entre adversarios que comparten referencias culturales. De la misma forma, no todos los conflictos llevan a un desenlace violento, aunque pueden incluir algunos aspectos de la violencia. Si la violencia perdura, se instala; o si pierde toda capacidad de ser instrumental y no puede ser controlada y limitada, entonces degrada el conflicto y lo lleva a otras lógicas de ruptura. El conflicto, aún cuando llega a sus formas más críticas, no impide la coexistencia de llegar a los acuerdos o las moderaciones, y es ahí en donde se explica y se entiende que ambos fenómenos -los acuerdos y conflictos- forman parte del carácter complejo de las relaciones sociales.

En ese sentido, el conflicto puede permanecer en el tiempo como una forma de regulación social, y como una manera de producir reglas y normas para un determinado espacio (Marié, 2004). Entendiendo que el conflicto también se presenta de manera modular, como lo plantean Panfichi y Coronel (2011), alternando momentos críticos con estados de latencia, y tomando distintas formas. En todo caso, puede mostrarse como relaciones de disputas que tienen como eje permanente la fricción social (Mejía, 2013).

Algo que parece importante resaltar es que Hoffmann (2009) propone tomar en cuenta la heterogeneidad económica, social y cultural de las sociedades en conflicto. Eso nos permite explicar procesos conflictivos, que muchas veces suelen entenderse en términos de antagonismos entre grupos, cuando en realidad traducen y revelan combinaciones más complejas de intereses y posturas.

Lo anterior ayuda a no perpetuar el conflicto vinculándolo con un solo hecho, sino a ver las modalidades de negociación y adaptación multifacética entre varias lógicas y recomposiciones que interactúan a distintos niveles (Hoffmann, 2009). Es así como las negociaciones surgen de las alianzas que históricamente han estado presentes, o de las relaciones de reciprocidad que se establecen cuando se comparten territorios.

Es el caso de la frontera México-Guatemala, que si bien se conformó en el campo del conflicto por la delimitación geopolítica, la continuidad social correspondiente a las dinámicas de las fuerzas regionales a la que refiere Castillo (1999) se impuso sobre los intereses y capacidades de los poderes centrales. Es así como en los hechos los intercambios y los vínculos entre los pueblos vecinos fueron cimentando una noción de región fronteriza. Por supuesto, eso no quiere decir que en estos escenarios transfronterizos locales no se reproduzcan las relaciones hegemónicas que se presentan en los Estados nacionales entre México y Guatemala. A continuación se describe la conformación de la frontera en esos contextos.

La conformación de la frontera geopolítica entre México y Guatemala 
6 Acuerdos y conflictos en dos localidades fronterizas Chuj entre México y Guatemala Mejía González, L.

El territorio fronterizo que comparten México y Guatemala se conformó en el campo del conflicto, en las relaciones de poder y en la implementación de las políticas colonizadoras del Estado nacional mexicano (Cruz, 1998; De Vos, 1993 y 2005; Hernández, 2001; Limón, 2007, 2008).

El acondicionamiento del territorio fronterizo estuvo sujeto a los grupos de poder que se movían en los ámbitos regional, nacional e internacional. De Vos (1993) señala que estos grupos actuaron, decidieron e incidieron lejos de las líneas que habían establecido, mientras que los pobladores que se quedaron en la zona de la frontera consideraron odiosa esta línea, debido a que era vista como un proyecto de expansión, ideado y ejecutado por unos pocos, en detrimento de muchos.

Esta frontera se explica jurídicamente con la conformación del Estado nación, y es fortalecida mediante la colonización de los espacios que se consideraban "vacíos". Por lo tanto, se podía observar que el poder estatal era al mismo tiempo individualizante y totalizante, como se demostró con la implementación del proyecto nacional homogeneizador y centralista que el Estado mexicano reforzó durante el siglo XX.

Estas estrategias políticas que México implementó para el fortalecimiento de la frontera y la colonización de la misma, permearon las relaciones entre las comunidades vecinas de ambos países. Comenzaron a emerger las desigualdades entre los pobladores de un país y de otro. El Estado mexicano organizó el territorio, la tierra, el agua y las relaciones sociales del lado mexicano, pero esta acción trajo consecuencias negativas para los vecinos del lado guatemalteco.

Con la división y el tratado de la frontera que se dio en 1882, Guatemala perdió una gran extensión territorial: 14 pueblos, 19 aldeas y 54 rancherías con más de 15000 habitantes pasaron a formar parte de territorio mexicano, mientras que México cedió a Guatemala un solo pueblo y 28 rancherías, con 2500 habitantes. Además, gran parte de la Selva Lacandona pasó a manos del gobierno mexicano. Esta pérdida fue resentida por el gobierno guatemalteco, pues se trataba de una zona casi despoblada, pero rica en maderas preciosas (De Vos, 1988 y 1993).

Durante un largo periodo, México y Guatemala sufrieron una serie de conflictos por sus territorios. El tratado de límites ponía en desventaja al gobierno guatemalteco debido a la pérdida de sus tierras y a la par, porque se presentaban disputas entre las empresas madereras que poseían concesiones en la franja fronteriza (De Vos, 1988). Fue hasta 1895 cuando se puso fin al proceso de la conformación de la frontera nacional, y a partir de ese año la línea divisoria internacional no sufrió más cambios. No obstante, la construcción de este artificio fronterizo influyó en la vida de la gente que quedó afectada por el establecimiento de las barreras políticas. De Vos (1993) menciona que las comunidades campesinas, en su mayoría indígenas, fueron afectadas porque pasaron a ser parte de lugares periféricos, fronterizos, cuando se habían considerado desde tiempos inmemoriales como parte de regiones céntricas. 
A partir del tratado de límites, se separaron los Estados geográfica y administrativamente y con ello, también una serie de pueblos y comunidades. A pesar de su cercanía, la frontera política marcó un camino para diferenciarlos en uno u otro país.

Aunque también Guatemala estaba viviendo un periodo de reforma liberal para finales del siglo XIX, Fábregas Puig (1992) menciona que esta reforma trajo como consecuencia diversos cambios, particularmente en la tenencia de la tierra, lo que dejó desarraigados a grupos étnicos y por lo tanto, tuvieron que cruzar la frontera política en 1886.

Una vez declarada la frontera límite, en 1883 el gobierno mexicano, bajo el mandato de Porfirio Díaz, decretó la Ley de Colonización de los Terrenos Nacionales, la cual permitió la nacionalización de los grupos indígenas, entre los que se encontraba el grupo chuj de Guatemala, asentado en las orillas del Sistema Lacustre de Montebello (Cruz, 1998). Esta frontera en términos del Estado nacional dejó al poblado de Tziscao del lado mexicano, y lo "separó" de la aldea vecina de El Quetzal, quedando esta última en territorio guatemalteco.

\section{Tziscao y El Quetzal: cercanos y distantes de la frontera}

En los estudios de Cruz (1998) y Limón (2007 y 2008) se afirma que los pobladores de Tziscao se establecieron a partir de la Reforma Liberal de Guatemala, en los años setenta del siglo XIX. Limón (2007) menciona que se dio una diáspora entre los chujes de San Mateo Ixtatán, y en ese contexto se fundaron varias localidades como Subajasum, Bulej y Tziscao, que se ubicaron en la ladera del norponiente de San Mateo hacia la frontera, que en ese momento estaba indefinida. Los pobladores que vivían en San Mateo Ixtatán buscaron nuevos asentamientos porque la tierra aún era libre; las familias comenzaron a adquirir lugares donde obtuvieron mayores productos de sus cultivos, y así se conformaron varias aldeas.

Por su parte, Cruz (1998) señala que los primeros pobladores de la zona llegaron en búsqueda de tierras para vivir debido a los conflictos que se estaban presentando en sus tierras de origen. Con la Reforma Liberal de Guatemala y la llegada del gobierno de Rufino Barrios en 1873 , se presentó un decreto sobre las tierras que establecía que estas no podían "titularse", y esos terrenos pasaron a manos de grandes terratenientes para el cultivo del café. Al mismo tiempo, se había dictaminado un Reglamento de Jornaleros (1877) y una Ley contra la Vagancia (1878) para tener segura la mano de obra indígena. Esta adquisición de tierras por grupos terratenientes llevó a la fundación de nuevos centros de población de grupos ladinos en territorios chuj, lo cual provocó la migración de pueblos nativos hacia lo que después serían “tierras mexicanas". Piedrasanta (2009) afirma que los ladinos jugaron un papel importante para poner en marcha un gobierno nacional en Guatemala, y su participación llevó a una nueva configuración espacial a escala regional y nacional. Los ladinos aseguraron los cargos político-administrativos más importantes, principalmente en las periferias, en las que más tarde se colonizaría la frontera. 
En la zona noroccidental de Guatemala, el proceso de colonización de las tierras baldías estuvo acompañado de una política de contrarreforma agraria de los años sesenta y setenta del siglo XX. Las zonas despobladas y los terrenos ociosos propiedad de la nación fueron declarados como área de desarrollo agrario. Sin embargo, las propiedades privadas no fueron objetos de expropiación. El objetivo de la colonización por parte del Estado guatemalteco llevó al desplazamiento de grandes grupos campesinos e indígenas a lugares lejanos, sin medios de comunicación y sin ningún tipo de servicio. Para el gobierno guatemalteco era urgente generar una barrera humana para resguardar la frontera (Piedrasanta, 2009).

En la tradición oral de los ancianos del lugar, Tziscao fue fundado en 1870 por un grupo de familias chuj que llegaron de diferentes aldeas cercanas a San Mateo Ixtatán de la República de Guatemala, y por familias que vivían cerca del municipio de La Trinitaria, en Chiapas. No obstante, con el tratado de límites entre México y Guatemala de 1882, Tziscao fue uno de los poblados que pasó a formar parte del territorio mexicano.

\section{Mapa 2. Zona fronteriza estudiada}

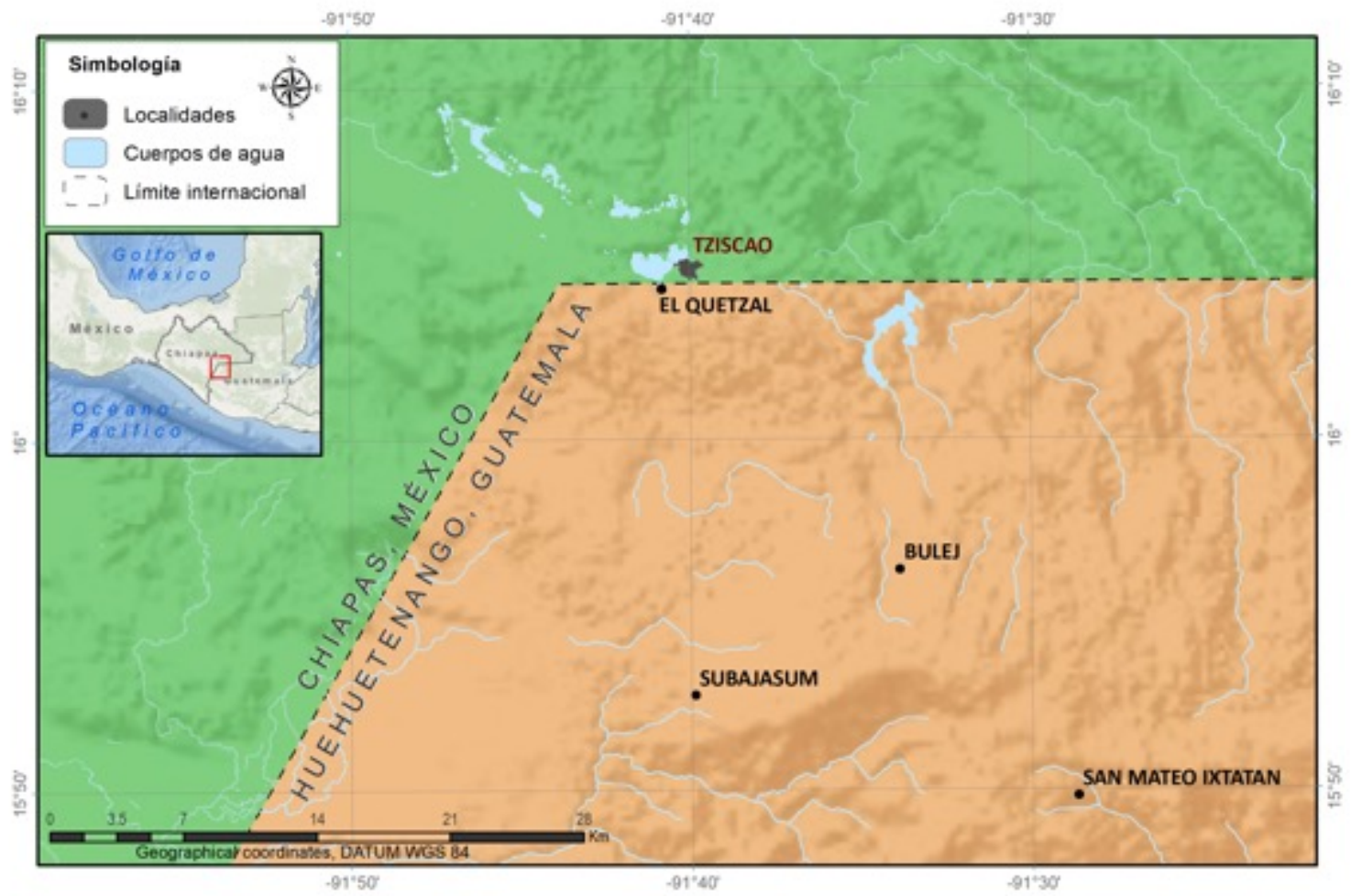

Fuente: Capas temáticas del Instituto Nacional de Estadística y Geografia (Inegi, 2010) y del Ministerio de Agricultura, Ganadería y Alimentación (MAGA, 2005). Elaboró: César Octavio Sánchez Garay.

A partir de la firma del Tratado de límites entre México y Guatemala en 1882, la región quedó integrada legalmente al territorio mexicano. Un año más tarde, se decretó la Ley de Colonización de Terrenos Nacionales, que "mexicanizó" a los indígenas chuj que habitaban la comunidad de Tziscao (Mejía y Peña, 2015). 
Con los límites fronterizos marcados legalmente y como resultado de los procesos de colonización, diez familias de Tziscao lograron obtener documentos oficiales de la propiedad de la tierra en la que se habían asentado. En las tierras oficiales comenzaron a cultivar maíz, frijol y calabaza, principalmente. Su cercanía con la laguna les permitió realizar actividades de pesca para sustentar y enriquecer su alimentación. La pertenencia al lugar se fue construyendo a medida que el grupo iba creciendo, pues en su interior comenzaron a darse nuevas relaciones de comercio y de administración que le permitía moverse a las cabeceras municipales de Comitán y La Trinitaria, en Chiapas, México. Al mismo tiempo, algunos integrantes de esta colonia seguían siendo trabajadores temporales de fincas cafetaleras cercanas a la selva chiapaneca en territorio mexicano, a las cuales se trasladaban en las temporadas de cosecha.

Las diez familias que en ese momento vivían en las orillas de la laguna Tziscao fueron dotadas de títulos de propiedad y de las tierras comunales denominadas El Ocotal. Con estos documentos, los habitantes obtuvieron el derecho de uso y aprovechamiento de las tierras, las montañas, las lagunas, y todos los recursos naturales que tenían a su alcance para vivir.

Sin embargo, Tziscao, ubicado al interior del sistema lagunar de Montebello en 1959, fue incluido en un polígono de casi 6500 hectáreas, decretado como Parque Nacional Lagunas de Montebello, y formalmente fue sometido a un control político-administrativo a cargo de la entonces Secretaría de Agricultura y Ganadería.

Para los habitantes de Tziscao, la tierra era su principal patrimonio y sustento de vida familiar, pero su dinámica cotidiana no dejaba de estar regida por los cambios que se daban a escala nacional, como las políticas del gobierno federal mexicano relacionadas con las formas de poblamiento, el reparto de tierras y el cambio de límites en los territorios.

El hecho de asegurar que los grupos indígenas chuj obtuvieran tierras de uso común y una "identidad nacional mexicana", provocaba marcadas diferencias. Mientras a los pobladores de Tziscao se les dio el derecho de poseer tierras comunales, y posteriormente ejidales, los que se quedaron del otro lado de la frontera seguían siendo trabajadores acasillados de las fincas cafetaleras, ubicadas en las tierras en las que actualmente ocupan como establecimiento poblacional; estas familias continuaron siendo trabajadoras jornaleras de la finca denominada Maber, hoy reconocida oficialmente en el censo poblacional de Guatemala como finca El Quetzal.

Las familias chuj que se quedaron del lado guatemalteco también sufrieron fuertemente el conflicto armado interno que vivió Guatemala durante los años ochenta del siglo XX, y buscaron refugio del lado mexicano. La mayoría de estas familias se asentaron en campamentos y en casas de familias cercanas a la línea fronteriza. La cercanía geográfica con México salvó la vida de muchas familias y comunidades, alejándolas de persecuciones y agresiones que vivían en sus propios lugares. La buena recepción de los refugiados guatemaltecos por comunidades mexicanas se debió a las relaciones históricas de pueblos vecinos, entre grupos étnicos y por afinidades socioculturales (Castillo, 1994). 
A partir de la década de los noventa, se dio el retorno de muchas de estas familias hacia Guatemala; a pesar de la fragmentación social y haber vivido un periodo de grandes violencias, muchas retornaron y comenzaron nuevamente con la conformación de sus aldeas. Con el tiempo, las familias de El Quetzal lucharon y pagaron para ser dueños de las tierras.

Actualmente, los habitantes de estas poblaciones alejadas de la capital tienen que recorrer caminos de terracería y utilizar transporte público durante aproximadamente 18 horas para llegar a la Ciudad de Guatemala. Y por ejemplo Nentón, cabecera municipal del departamento de Huehuetenango, está a dos horas de El Quetzal, y el transporte público para trasladarse no es constante. Esta lejanía de los centros administrativos, comerciales y económicos, aunada a la falta de servicios públicos de salud y energía eléctrica, ha llevado a la población de la aldea a negociar, acordar y convenir con la población de Tziscao su paso por la frontera y otros servicios. Las familias de El Quetzal necesitan cruzar a la comunidad de Tziscao, del lado mexicano, para transitar hacia la carretera federal que las lleva a las cabeceras municipales de Comitán y La Trinitaria.

A las localidades de El Quetzal y Tziscao las separa la línea fronteriza internacional. Sin embargo, ambas comparten una identidad chuj y un territorio lleno de agua, que se ha convertido en un lugar turístico de gran importancia en los últimos años.

Fotografía 1. Laguna Internacional México-Guatemala

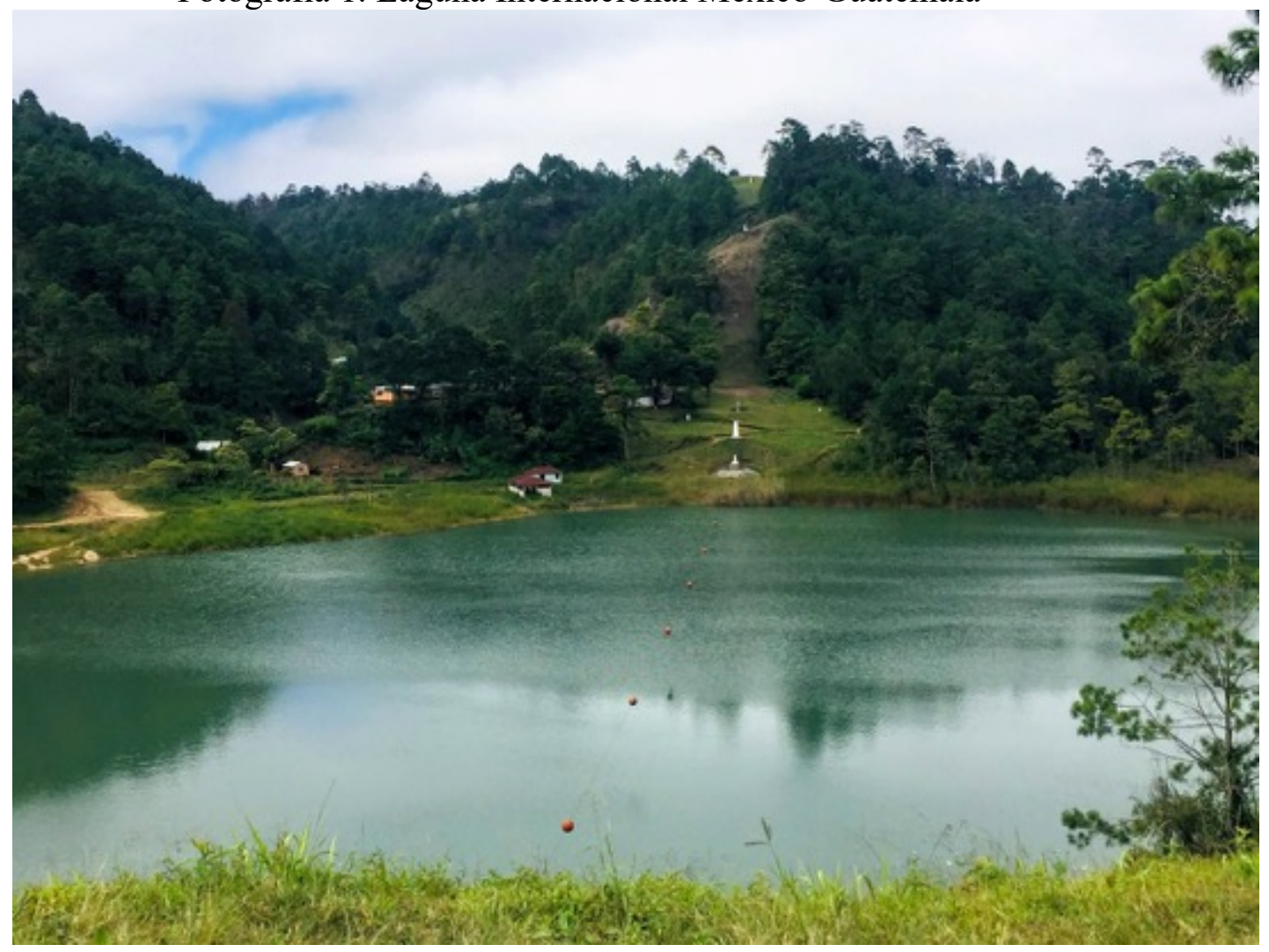

Fuente: Sobre el lago se aprecia la división internacional de la laguna ubicada entre Tziscao y El Quetzal, México-Guatemala. Fotografía tomada por la autora. 
Vivir a orillas de la laguna Tziscao y del llamado lago Binacional les ha permitido a los pobladores de ambas comunidades realizar diversas actividades como la pesca o las actividades recreativas; el agua es usada para uso doméstico y en algunos momentos fue utilizada para consumo humano; el hecho de ser una zona de tierras húmedas y fértiles les permite tener zonas de agricultura para la siembra de maíz, frijol y café.

El turismo y el comercio han sido dos actividades que llegaron para quedarse; entre estos sectores se desenvuelve la vida cotidiana de mujeres, hombres, niñas y niños, que diversifican sus actividades en la venta de comida o de artesanías que se ofrecen en los paradores turísticos, así como de guías de turistas, balseros, responsables de casetas de cobro y de transporte local. En esta zona, además de ofrecer la belleza natural del sistema lacustre, el límite fronterizo forma parte del paisaje.

\section{Comunidades vecinas: entre acuerdos y conflictos}

Vivir en la frontera también implica negociar el paso. Aunque ambas comunidades tienen libre tránsito para cruzar de un lugar a otro debido a que no existe un puesto de control migratorio, los pobladores de El Quetzal suelen ser los más desfavorecidos, ya que se ven en la necesidad de atravesar por terrenos que pertenecen a la comunidad de Tziscao para llegar a la carretera fronteriza que conecta con el resto del territorio mexicano. Es un tramo de dos kilómetros, conformado por un camino de terracería que después se convierte en una de las calles principales de la localidad de Tziscao.

Para transitar por ese tramo carretero del lado mexicano, las autoridades de Tziscao consideraron que debían compartir responsabilidades con los pobladores de El Quetzal para el mantenimiento de la carretera, que no era utilizada únicamente por los lugareños, sino también por el turismo. Fue así como en 2008 se estableció un convenio que consistía en reparar el tramo de terracería por parte de los aldeanos de El Quetzal; a cambio, algunas familias de Tziscao obtendrían agua para uso doméstico, que llevarían a través de un sistema de agua rodada desde los manantiales de El Quetzal, ubicados en territorio guatemalteco. En este acuerdo también consideraron que las familias de El Quetzal podían hacer uso de los servicios de salud y de educación de Tziscao, aunque de alguna manera los aldeanos ya utilizaban algunos de estos servicios.

La población de Tziscao, a pesar de contar con la laguna más extensa, siempre enfrentó el problema de no contar con agua entubada en sus viviendas, y ha tenido que hacer uso de las lagunas, manantiales y arroyos que cruzan por la comunidad, a través del acarreo de agua por medio de cántaros. 
12 Acuerdos y conflictos en dos localidades fronterizas Chuj entre México y Guatemala Mejía González, L.

Fotografía 2. Acarreo de agua para uso doméstico en Tziscao

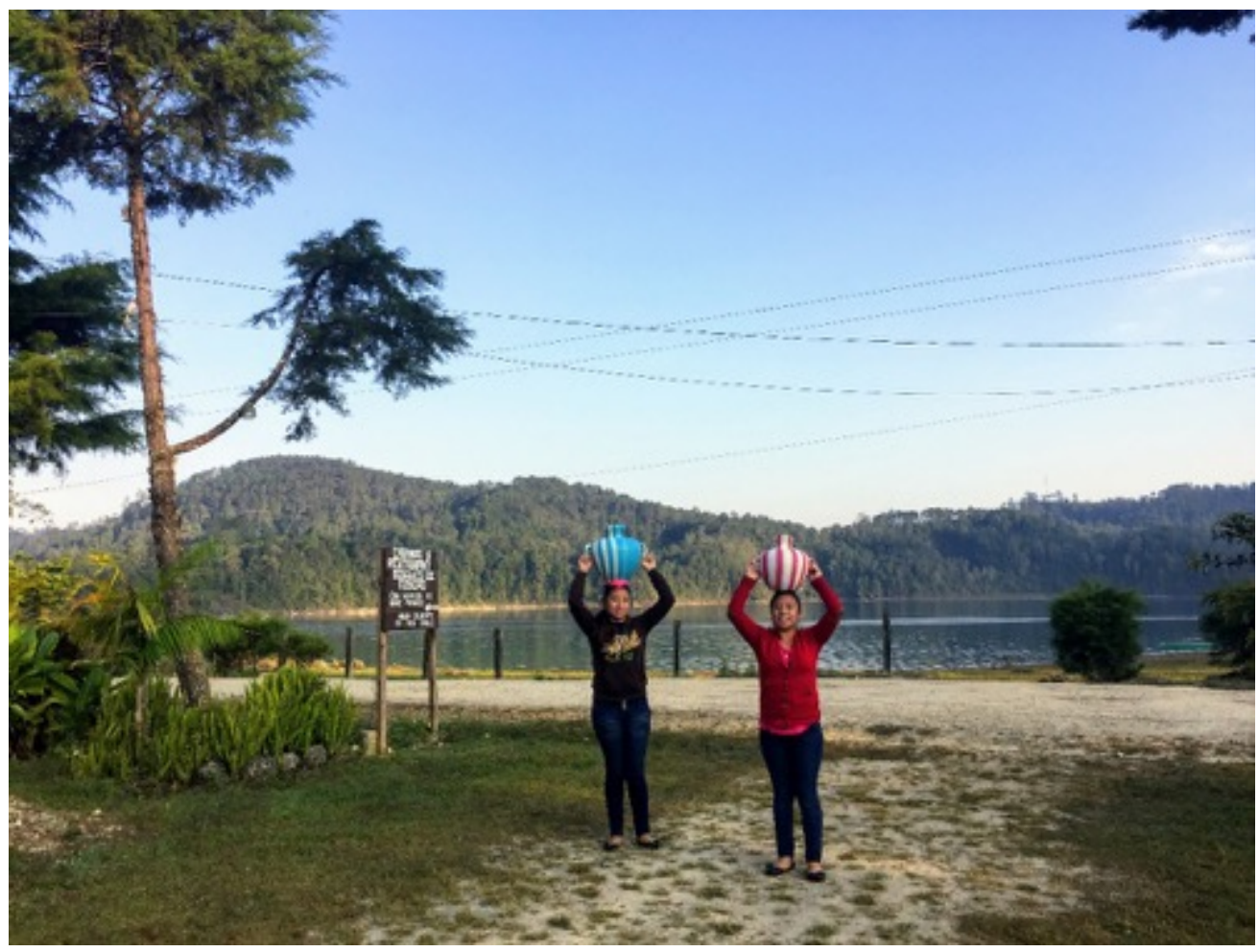

Fuente: Tziscao, Chiapas, México. Fotografía tomada por la autora.

Con el convenio de libre tránsito, las autoridades de Tziscao construyeron un tanque de almacenamiento de agua en terrenos que corresponden a El Quetzal, y otro tanque del lado mexicano. De ahí se distribuía el agua hacia el Parador Internacional y hacia algunas viviendas que estaban en la parte más baja de la localidad; este sistema no cubría en su totalidad a las viviendas, y muchas de las familias continuaron con el acarreo de agua.

Los acuerdos que se habían realizado a través de las autoridades de ambas localidades comenzaron a fragmentarse a finales de 2010, cuando pobladores de El Quetzal cortaron la manguera y evitaron que llegara el agua a los tanques de almacenamiento. Este hecho desató una serie de tensiones entre la población de Tziscao. La versión de los aldeanos fue que se debió a un ajuste de cuentas debido a que las autoridades de Tziscao les estaban cobrando el paso, a pesar de la rehabilitación del camino que ellos hacían año con año. La población de El Quetzal se negó a pagar la cantidad de 8000 pesos que les pedían las autoridades de Tziscao, y en respuesta a su molestia, decidieron cortar las mangueras que llevaba el agua a las viviendas.

Ambas partes tenían su propia postura y la defendían sobre sus derechos. Por supuesto, a pesar de eso los pobladores de Tziscao no pudieron incidir en el cierre del paso fronterizo, 
porque también son beneficiados por el turismo que cruza hacia Guatemala. Además, en los últimos años han tenido la presencia del turista guatemalteco que atraviesa la línea fronteriza para visitar el conjunto de lagunas de lado mexicano.

La frontera se volvió más dinámica con el aumento del turismo a partir del año 2010, principalmente de nacionales y regionales. No obstante, los visitantes prefieren comprar artesanías cuando cruzan a Guatemala, y no del lado mexicano, que también ofrece los mismos tipos de souvenirs. Esto último ha sido otro factor por el cual las tensiones se hacen cada vez más fuertes entre ambas comunidades. Actualmente los negocios de las mujeres de Tziscao - que por años han vendido artesanías del país vecino-, han disminuido debido a la competencia de los comercios que tienen las familias de El Quetzal, al otro lado de la frontera.

El paso por la frontera ha sido un tema conflictivo, que se ha agudizado en los últimos años. El 1 marzo del 2012 amaneció un aviso informativo que las autoridades de Tziscao habían colocado junto a una caseta de cobro, a la salida rumbo a la aldea El Quetzal, en el que anunciaban que a partir de esa fecha el paso de la frontera tendría horarios de entrada y de salida. Además, habían colocado una cadena que permitía cerrar literalmente la carretera.

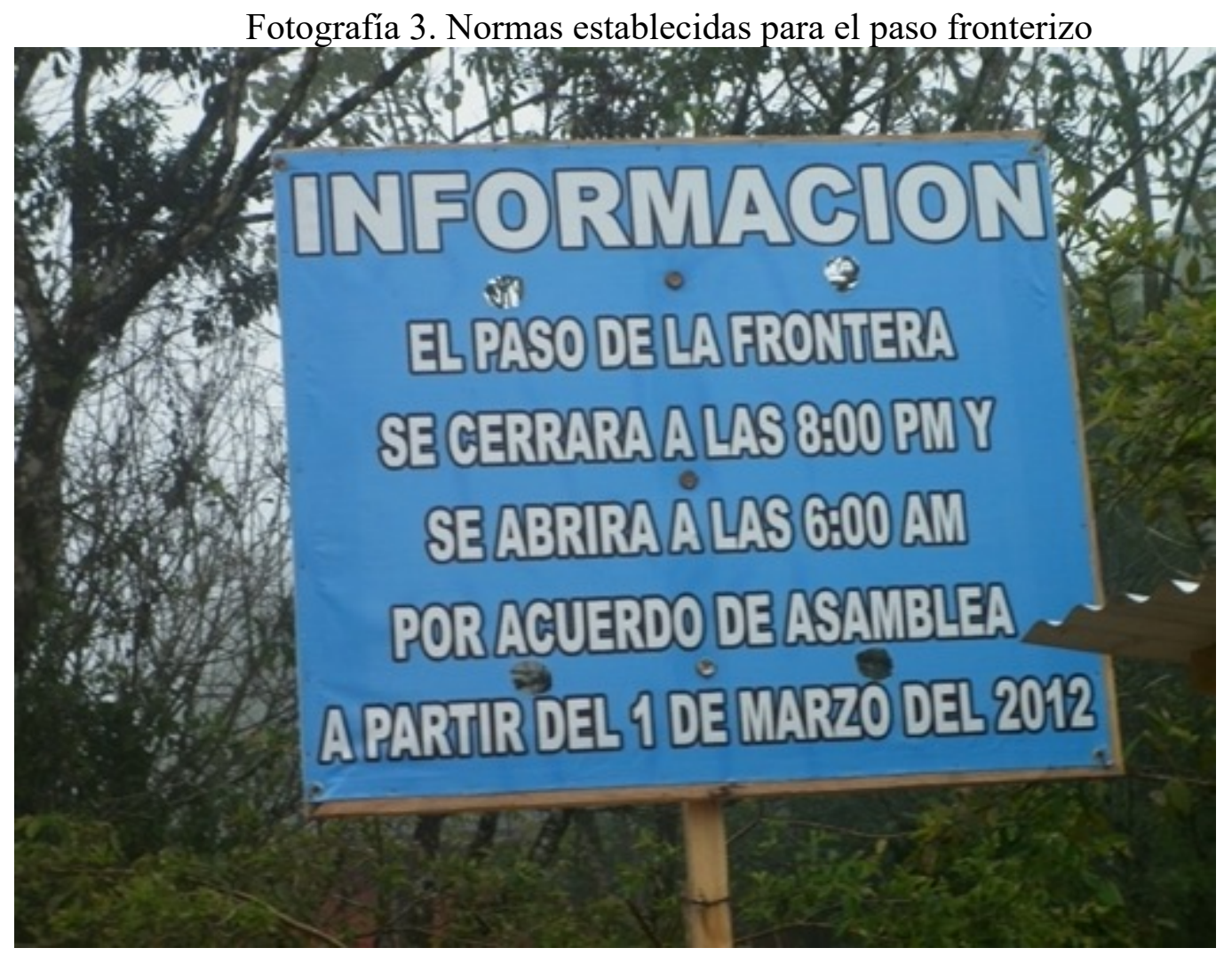

Fuente: Tziscao, Chiapas, México. Fotografía tomada por la autora.

La asamblea ejidal de Tziscao había acordado cobrar el paso de vehículos pertenecientes no únicamente de las personas que vivían en El Quetzal, sino para todos los que transitaban de Guatemala hacia México. Además, era una forma de cobrar la falta de cumplimento de 
14 Acuerdos y conflictos en dos localidades fronterizas Chuj entre México y Guatemala Mejía González, L.

los aldeanos del otro lado, que ya no estaban rehabilitando el tramo carretero, ni dotando de agua a la población de Tziscao.

Las tarifas de pago se diferenciaban; para los vehículos que provenían de El Quetzal e iban hacia la carretera federal fronteriza del lado mexicano, el costo era de 30 pesos, y para los que iban de otras localidades guatemaltecas, dependía del tipo de vehículo y lo que transportaban, algunos llegando a pagar hasta 150 pesos. Esta forma de cobro se convirtió en un tema que causó mucha molestia entre los pobladores de la aldea, pues lo referían como un acto de injusticia por parte de sus vecinos mexicanos, porque era un hecho que los pobladores de El Quetzal no tenían otras opciones de tránsito.

La población de Tziscao consideró que la mejor decisión era "dejar pasar" libremente por unos meses, para que la situación se apaciguara, sabiendo que la frontera era un espacio peligroso por el que podían pasar grupos de migrantes y vehículos con cargamentos que ellos desconocían. Las personas que viven a orillas de la carretera podían observar cómo Tziscao se había tornado en un lugar muy transitado en los últimos 10 años, debido a la apertura y la rehabilitación de la carretera de terracería que comunica a El Quetzal con otros pueblos, aldeas y municipios de Guatemala. Pero este tránsito se daba con mayor frecuencia en horarios de tarde y noche, y fue por eso que posteriormente la frontera se cerraba por la noche y abría su paso por la mañana.

Las medidas de regulación que adoptaba la asamblea ejidal para el paso fronterizo estaban permeadas por una serie de situaciones que enfrentaban como comunidades vecinas, y no se debían solo a un ajuste de cuentas y a la falta de un acuerdo por el agua. El turismo y el comercio también se habían convertido en factores de tensión; ambas comunidades querían ganar día con día mayor número de visitantes en sus lugares, para así obtener mayores ingresos, aunque son los ejidatarios de Tziscao los que tienen una fuerte organización en torno al turismo, y gran parte de las tareas cotidianas que realizan hombres y mujeres están relacionadas con esta actividad, por ejemplo, los balseros que llevan a cabo recorridos por las lagunas, guías de turistas, servicios de comida en los paradores turísticos y el complejo de cabañas administradas por los ejidatarios. A ello se le suma que muchas familias tienen negocios particulares de restaurantes, hospedaje y venta de artesanías.

Por supuesto que hay una marcada diferenciación de ingresos, mientras los pobladores de Tziscao ofrecen todos los servicios mencionados en el párrafo anterior, los aldeanos de El Quetzal se dedican únicamente a la venta de artesanías y de otros productos elaborados con cardamomo, café y cacao. Pese a estas diferencias, los aldeanos de este último lugar tienen la ventaja de estar del lado guatemalteco, porque muchos turistas llegan a la línea, cruzan la frontera y adquieren ahí sus productos.

La actividad turística como competencia les dio a las familias de El Quetzal la fortaleza para tomar decisiones, aunque les trajo mayores consecuencias. Por ejemplo, en octubre de 2012 las autoridades de El Quetzal habían logrado hacer la compra-venta del terreno donde 
se transita hacia la aldea, el camino de terracería que forma parte de la comunidad de Tziscao, es decir, en terrenos mexicanos.

La venta de este pedazo de tierra no fue avalada por las autoridades ejidales de Tziscao, pues el arreglo se llevó a cabo entre el dueño del terreno y las autoridades de la aldea de El Quetzal. Como "dueños", los de El Quetzal decidieron rehabilitar y asfaltar un tramo de aproximadamente 400 metros. Cuando la obra estaba terminada, las autoridades de Tziscao cerraron definitivamente el paso para los vehículos que cruzaban la frontera por ese camino, obstruyendo el inicio y el final de la vía con grandes zanjones y posteriormente, con la construcción de un muro encementado que intentaba disimular paisajísticamente el conflicto.

Fotografía 4 y 5. El cierre de la frontera entre El Quetzal y Tziscao
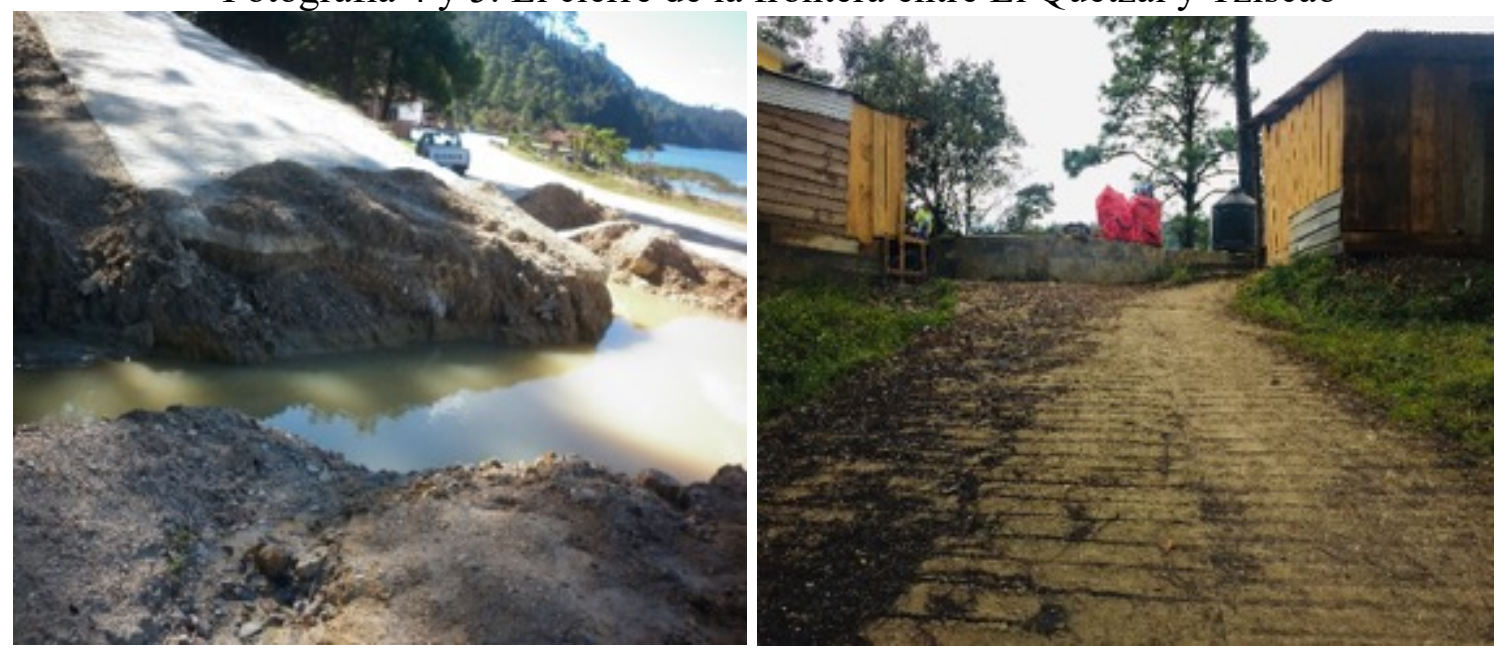

Fuente: Zanjas y muro que corta la vía construida por las autoridades de El Quetzal en terrenos de Tziscao, Chiapas, México. Fotografías tomadas por la autora.

Este hecho marcó una distancia entre los vecinos fronterizos; el límite geopolítico se reforzó cuando los aldeanos de El Quetzal recurrieron a las autoridades del consulado guatemalteco para que intervinieran en el conflicto. Sin embargo, las autoridades no lograron incidir en esta problemática. La Comisión Internacional de Límites y Aguas consideró este conflicto como un asunto local, que las poblaciones debían resolver. Además, la población de Tziscao no solamente validó la frontera nacional argumentando que al tratarse de otro país, los del El Quetzal no podían construir ninguna obra, sino que ratificó una frontera de conservación como bandera de defensa, con el argumento que Tziscao se encuentra al interior de un Parque Nacional, donde está prohibido construir caminos encementados.

La construcción de este muro para interrumpir la vía reafirmó la frontera nacional de los mexicanos frente a sus vecinos chuj guatemaltecos. Actualmente, se pueden observar las banderas de cada uno de los países, que ondean en el límite fronterizo como una forma de expresar una identidad nacional. Obstaculizar esta carretera afectó a los aldeanos en su vida cotidiana; tan solo por poner dos ejemplos, los pobladores de El Quetzal tienen que transportar sus productos que compran del lado mexicano sin utilizar vehículos, y trasladarlos 
a manera de carga, en la espalda; lo otro tiene que ver con lo dificultoso que se ha convertido llevar a un enfermo a unidades médicas del lado mexicano.

Las relaciones conflictivas no desaparecen, están siempre presentes en el día a día como tensiones o fricciones. Por supuesto, muchas veces se disimulan para continuar con las actividades de turismo. Además, continúan la cercanía geográfica, las relaciones familiares, de trabajo, y las actividades religiosas. En ese contexto, se puede observar que el límite no está en cada persona que lo transita, pues no es un asunto individual, y la frontera nacional se colectiviza.

\section{Relaciones de parentesco, redes familiares y de trabajo}

Las relaciones socioculturales, de parentesco y de trabajo, principalmente, son las que de alguna manera apaciguan los conflictos que se presentan entre El Quetzal y Tziscao. Por ejemplo, existen relaciones entre padres e hijos, hermanos y hermanas, de compadrazgo y de amistad; o matrimonios que continúan celebrándose a pesar del decreto fronterizo. En la cotidianidad, se pueden observar reuniones familiares de integrantes de cada una de las comunidades, visitas familiares de uno y otro lado de la frontera, celebraciones como fiestas de cumpleaños y bodas. En la fiesta religiosa de la Virgen de Candelaria, que se celebra el 2 de febrero en Tziscao, las familias y los jóvenes de El Quetzal asisten a la ceremonia y al baile que se realiza por la noche.

En las relaciones de parentesco no hay reglas o normas que acordar, pero sí existen ciertas consideraciones en la conformación de los matrimonios, sobre todo en las familias de Tziscao. Los padres prefieren que sus hijas se casen con hombres de la misma comunidad, pues consideran que tienen mejores condiciones de vida, mientras que los hijos varones son los que adquieren la herencia de los derechos ejidales, que incluyen la tierra y actividades turísticas, y que les permite asegurar la continuidad de sus generaciones.

Los hombres mexicanos de Tziscao pueden casarse con mujeres fuereñas, mexicanas o guatemaltecas, y llevarlas a vivir allí. Sin embargo, las mujeres mexicanas de Tziscao que se casan con hombres de otros lugares, mexicanos o guatemaltecos, obligatoriamente tienen que salir de su grupo familiar y de la comunidad debido a que el esposo no puede llegar a residir en tierras ejidales, y mucho menos obtener el derecho ejidal y de ejercer actividades turísticas. Actualmente, el ejercicio matrimonial en Tziscao permite ver cómo los derechos están condicionados por normas y reglas que se establecen por y para el derecho de la tierra y la organización del turismo.

Otra de las relaciones fuertes que existen entre Tziscao y El Quetzal se da en el sector de servicios, conformado en su mayoría por mujeres de El Quetzal, contratadas para trabajar en los restaurantes, paradores turísticos y cabañas de las familias de Tziscao. Ellas realizan labores como la preparación de tortillas, el lavado de la ropa, la limpieza de las habitaciones de las cabañas y/o ayudantes de la cocina. Todos los días las mujeres guatemaltecas que se emplean caminan más de 500 metros para estar del lado mexicano, y por las tardes, al 
terminar su jornada de trabajo, retornan a la aldea para continuar con sus tareas familiares; los contratos para estas actividades no son permanentes, pues dependen de la temporada alta de turismo, aunque en los últimos años muchas mujeres de El Quetzal se emplean en su mismo lugar de origen, apoyando en la venta de artesanías y diversificando las actividades que se ofrecen al turismo.

En cuanto a los guías de turismo local de Tziscao, estos realizan arreglos con los dueños de negocios de artesanías de la aldea de El Quetzal. Por ejemplo, existe un acuerdo de pago que se denomina "por comisiones", y que consiste en que los grupos de turistas son llevados por los guías locales a las casas de artesanías de la aldea vecina; por supuesto, el pago depende del número de turistas y de la compra que ellos realicen.

Una situación importante de intercambio que debe resaltarse entre ambas comunidades son los medios de comunicación, específicamente, el servicio de telefonía celular. La población del ejido Tziscao logró tener la cobertura de este servicio por parte la empresa guatemalteca Tigo, ${ }^{2}$ cuya cobertura abarca una parte del lado mexicano. Ambas comunidades -El Quetzal y Tziscao, y muchas otras comunidades de la frontera- comparten la misma clave lada. La población de Tziscao recurre a los pequeños establecimientos de la aldea vecina para realizar recargas telefónicas, aunque en Tziscao también existen dos establecimientos pequeños de Tigo, que sirven para la compra y recarga de tiempo aire. La comunidad de Tziscao no ha tenido cobertura de telefonía celular por alguna empresa que opere en territorio mexicano, por lo tanto, para esta población realizar una llamada a cualquier parte del territorio mexicano implica una llamada internacional.

En las relaciones socioculturales, familiares, económicas y de comunicación se aprecian todos aquellos vínculos, como un mismo grupo chuj, que han estado presentes antes y después del decreto fronterizo. Los lazos familiares, de amistad o de compadrazgo funcionan como puentes que se tejen para permanecer en la cercanía, no sólo geográficamente, sino también como grupo que los identifica.

\section{CONCLUSIONES}

Entender los conflictos y acuerdos como parte de las relaciones sociales y desde los procesos históricos, como proponen Marié (2004), Wieviorka (2010) y Lederach (1992), lleva a otras discusiones y formas de mirar lo que sucede en las fronteras; no se trata de minimizar dichos acontecimientos, ni de pasar por alto que en los conflictos se presentan relaciones desiguales debido a la nacionalidad o a otros componentes económicos, políticos y culturales, como se ha podido leer en este caso de estudio. Para intentar ganar la partida, cada comunidad estudiada utiliza estratégicamente sus recursos: el agua, el límite fronterizo, su condición nacional, el turismo, el comercio.

${ }^{2}$ Esta telefonía corresponde a la marca de Millicom International Cellular S.A. (MIC) y opera en América Central, Sudamérica y África. 
Sin embargo, lo que se ha podido constatar en este caso es que muchas veces el conflicto toma diversas formas, y no siempre está en su punto más álgido y de violencia. El conflicto se oculta, se disimula frente al turista, porque lo que se pretende es mostrar un lugar de tranquilidad, de acuerdo con las características paisajísticas del Sistema Lagunar de Montebello. Pero siempre está ahí el roce cotidiano, la fricción social y la aceptación de las contradicciones.

Frente a estos contextos, también la frontera toma diferentes matices. Entre la distancia y la proximidad (Castillo, 1999), la frontera se vuelve flexible, móvil y de alguna manera, accesible; la frontera ofrece accesos diferenciados de bienes materiales y de servicios a partir de la condición nacional de sus habitantes y poblados; el componente de diferenciación que ha convertido a unos en usufructuarios de derecho pleno y a otros, en excluidos. También hay una frontera límite que marca la soberanía nacional entre mexicanos y guatemaltecos, visible por una historia en la que las decisiones y los intereses se escinden donde se dividen políticamente los países. Es ahí que se explican las tensiones estructurales a escalas nacionales, y la falta de cooperación entre ellas.

Ambas fronteras son el resultado de las decisiones que tomaron y toman grupos hegemónicos, y esto se reproduce a escala local. Dehouve (2001) plantea que las políticas administrativas establecidas por el Estado nacional están regidas por un principio de desigualdad entre las unidades territoriales, las cuales pueden determinar los conflictos y las dinámicas locales, en términos de competencia.

El caso entre Tziscao y El Quetzal es un ejemplo que muestra cómo el Estado mexicano y el guatemalteco han marcado una diferencia que se refleja en las decisiones que se toman al interior de cada comunidad hoy en día, ya sea para negociar el paso de la frontera, o para convenir y tener servicios de salud, agua o comunicación que los mismos gobiernos no pueden ofrecer en lugares periféricos como este. También se suman otros sectores, como el turismo y el comercio, que hacen que los lugares vecinos estén en competencia por mayores ingresos económicos. Sin embargo, algunos acuerdos, arreglos y relaciones socioculturales de continuidad e historias de reciprocidad, siguen siendo los puentes que sostienen las relaciones sociales en este fragmento fronterizo.

\section{REFERENCIAS}

Castillo, M.A. (1994). Chiapas: Escenario de conflicto y refugio. DemoS, (7), 25-26.

Castillo, M.A. (1999). La vecindad México-Guatemala: Una tensión entre proximidad y distancia. Estudios Demográficos y Urbanos, 14(1), 193-218. https://doi.org/10.24201/edu.v14i1.1041

Castillo, M.A. (2002). Introducción. Migraciones y movilidad espacial en la frontera sur. En E. Kauffer Michel (Ed.), Identidades, migraciones y género en la frontera Sur de 
México (pp. 187-192). San Cristóbal de Las Casas, Chiapas, México: El Colegio de la Frontera Sur.

Cruz, J.L. (1998). Identidades en Fronteras, Fronteras de Identidades. Elogio de la intensidad de los tiempos en los pueblos de la Frontera Sur. México: El Colegio de México.

De Vos, J. (1988). Oro Verde. La conquista de la Selva Lacandona por los madereros tabasqueños, 1822-1949. México: Fondo de Cultura Económica.

De Vos, J. (1993). Las fronteras de la frontera sur. México: Universidad Autónoma de Tabasco/Centro de Investigaciones y Estudios Superiores de Antropología Social.

De Vos, J. (2005). La formación de la frontera entre México y Centroamérica. México: El Colegio de la Frontera Sur.

Dehouve, D. (2001). Ensayo de geopolítica indígena. Los municipios tlapanecos. México: Miguel Ángel Porrúa/Centro de Investigaciones y Estudios Superiores en Antropología Social/Centro de Estudios Mexicanos y Centroamericanos.

Fábregas Puig, A. (1992). Pueblos y culturas de Chiapas. México: Gobierno del Estado de Chiapas/Miguel Ángel Porrúa.

Hernández, Castillo, R. A. (2001). La otra frontera. Identidades múltiples en Chiapas poscolonial. México: Centro de Investigaciones y Estudios Superiores en Antropología Social.

Hoffmann, O. (2009). Lugares de fronteras. Lecturas de un conflicto territorial en el sur de Veracruz, siglos XVIII y XIX. En E. Velázquez, E. Léonard, O. Hoffmann y M. F. Prévôt-Schapira (Coords). El istmo mexicano: Una región inasequible. Estado, poderes locales y dinámicas espaciales (Siglos XVI-XXI), (pp. 165-213). México: Centro de Investigaciones y Estudios Superiores en Antropología Social/Institut de Recherche Pour le Développement.

Instituto Nacional de Estadística y Geografía (Inegi). (2010). Censo de población y vivienda 2010. Recuperado de https://www.inegi.org.mx

Kauffer, E. F. (2011). Hidropolíticas en la frontera entre México, Guatemala y Belice: La necesaria redefinición de un concepto para analizar la complejidad de las relaciones en torno al agua en escenarios transfronterizos. Aqua-lac, 3(1), 157-166.

Lederach, J. P. (1992). Enredos, Pleitos y Problemas. Una guía práctica para ayudar a resolver conflictos. Guatemala: Ediciones Semilla.

Limón, F. (2007). Memoria y Esperanza en el pueblo Maya Chuj. Conocimiento cultural y diálogos en las fronteras. Puebla: Benemérita Universidad Autónoma de Puebla/Instituto de Ciencias Sociales y Humanidades Alfonzo Vélez Plieg. 
Limón, F. (2008). La ciudadanía del pueblo Chuj en México. Una dialéctica negativa de identidades. Alteridades, 18(35), 85-98.

Marié, M. (2004). Las huellas hidráulicas en el territorio. La experiencia francesa. San Luis Potosí, México: El Colegio de San Luis/Instituto Mexicano de Tecnología del Agua/ Secretaría de Medio Ambiente y Recursos Naturales.

Marié, M. (2015). Presentación: La construcción de los territorios en México. ¿Qué es un territorio hoy? En G. Santacruz y F. Peña (Coords.), Miradas sobre dinámicas territoriales en México, (pp. 9-20). México: El Colegio de San Luis.

Mejía, L. (2013). Reapropiación del territorio lacustre de Montebello: El caso de un pueblo fronterizo chuj en Chiapas. (Tesis doctoral), San Luis Potosí, México, El Colegio de San Luis, México.

Mejía, L. y Peña, F. (2015). Territorios disputados: Culturas y aprovechamiento de los Lagos de Montebello. En G. Santacruz y F. Peña (Coords.), Miradas sobre dinámicas territoriales en México, (pp. 839-59). México: El Colegio de San Luis.

Ministerio de Agricultura, Ganadería y Alimentación (MAGA). (2005). Atlas temático de la república de Guatemala (Serie de recursos naturales, sociales, productivos, amenazas y vulnerabilidad. Guatemala: Laboratorio SIG-MAGA.

Panfichi, A. y Coronel, O. (2011). Los Conflictos Hídricos en el Perú 2006-2010: Una lectura panorámica. En R. Boelens, L. Cremers y M. Zwarteveen (Eds.), Justicia Hídrica, acumulación, conflicto y acción social, (pp. 293-422). Perú: Justicia Hídrica/Instituto de Estudios Peruanos/Fondo Editorial Pontificia Universidad Católica del Perú.

Piedrasanta, R. (2009). Los Chuj, unidad y ruptura en su espacio. Guatemala: ARMAR.

Wieviorka. M. (2010). El conflicto social. Sociopedia.isa, (s/n), 1-10. Recuperado en http://www.sagepub.net/isa/resources/pdf/Social\%20Conflict\%20-\%20Spanish.pdf 